Journal of Sustainability Perspectives

journal homepage: https://ejournal2.undip.ac.id/index.php/isp/

\title{
An Innovative Approach to Sustainability
}

\author{
Okan Pala ${ }^{1, *}$ \\ ${ }^{1}$ Ozyegin University, Nisantepe Mah. Orman Sokak, Cekmekoy, Istanbul,Turkey \\ * corresponding author: okan.pala2@ozyegin.edu.tr
}

\section{Article Info}

\author{
Received: \\ 15 March 2021 \\ Accepted: \\ 25 May 2021 \\ Published: \\ 1 August 2021 \\ DOI:
}

Presented in The $6^{\text {th }}$ International (Virtual) Workshop on UI GreenMetric World University Rankings (IWGM 2020)

\begin{abstract}
Solutions to sustainable development (SD) challenges are complex and context-specific. Ozyegin University $(\mathrm{OzU})$ created a structure to manage all its sustainability endeavours aligned with these two facts. To this end, $\mathrm{OzU}$ first understood the priorities of Turkey to achieve SD in terms of SDGs. Subsequently, the university conducted a self-assessment to determine the SDGs that it is most competent at. By combining these two analyses, $\mathrm{OzU}$ determined the areas in which it can have the highest impact in the SD of Turkey. In conducting these analyses, a holistic approach was adopted, to consider the interconnectedness of the SDGs. The tools of the network analysis were employed to address the intertwined relationships among the SDGs. The analysis revealed four domains which led to the creation of four Sustainability Clusters. The clusters are positioned under the newly established Sustainability Platform managing and combining all the sustainability endeavors in relation with both these domains and otherwise. In this paper, the creation of processes of Sustainability Platform and Sustainability Clusters the structures which aim to contribute to the SD of Turkey will be elaborated. Practical insights will be developed based on this structure
\end{abstract}

\section{Keyword:}

sustainability management, multidisciplinarity, organizational structure, sustainability clusters

\section{Introduction}

The framework for SD is redefined with the introduction SDGs in 2015. The SDGs delineate a clear set of goals to be reached by 2030 in order to alleviate the grand problems. While deliberating her contribution to SD of Turkey, Ozyegin University (OzU) overcome two main challenges: i. the context-specificity of SD solutions [1]; ii. the interlinkages among the SDGs.

To address the locality of SD solutions OzU matched the needs of the context (i.e. Turkey) with its strengths. The needs of the context are made visible by various reports which $\mathrm{OzU}$ benefited from significantly. Subsequently $\mathrm{OzU}$ made a self-assessment 
understanding its own competencies regarding SDGs.

One of the challenges while conducting these analyses is the interdependent nature of the SDGs. For instance, [1] investigate the relationships between Sustainable Development Targets (SDTs) to attain the 2030 target for one of the SDGs, SDG 7 and show that 143 SDTs are in relationship with each other to reach SDG 7 targets. Therefore, attaining SDGs require high cross-disciplinarity. However, for instance in research, coordination among different disciplines can be challenging [2], [3]. There are various reasons for this failure such as scarcity of funding, a dearth of cooperation among departments and disciplines historically, differences in methodologies and disciplinary norms, turfism, and egos $[4,5,6$, 7, 8].

In order to overcome these obstacles and foster cross-disciplinarity Ozyegin University $(\mathrm{OzU})$ established Sustainability Clusters providing a unique organizational structure to manage SD initiatives. While forming these clusters, OzU first understood the priorities of Turkey to achieve SD in terms of SDGs including the interlinkages at the goal level. Subsequently, she conducted a self-assessment to determine the SDGs that she is most competent at. By combining these two analyses, $\mathrm{OzU}$ determined the areas in which she can have the highest contribution to the SD of Turkey.

In this paper, the approach of $\mathrm{OzU}$ to conduct cross-disciplinary research contributing to SD by addressing the obstacles in performing research in a cross-disciplinary manner will be outlined. Practical insights will be developed based on this unique approach.

\section{Sustainability Structure at $\mathrm{OzU}$}

\subsection{Determining the Contribution to SD: OzU's Competences, The Turkish Context, and Interlinkages Among the SDGs}

In order to determine the SDGs that the university can contribute the most, the intersection between the priorities of Turkey and the competences of $\mathrm{Oz} \mathrm{U}$ are intersected. To this end, initially the competencies of $\mathrm{OzU}$ had been determined. OzU has been founded as an entrepreneurial research university in 2007. Sustainability is one of the core values of the university and this value manifests itself in all the activities of the university including from operations to civic engagement as well as research and education. In all of these activities OzU takes SDGs as the basis. In order to determine the SDGs that OzU is competent at, all the projects (both research and implementation projects) to date have been evaluated on the basis of their contributions to SDGs. This process -that took almost a year- revealed that $\mathrm{OzU}$ has accumulated significant competencies in SDGs 5, 7, 8, 9, 10, and 11.

Second, the priorities of the context, i.e. Turkey, has been introduced to the analysis. To this end, the [9] report is employed. The report not only evaluates Turkey in terms of the situation of the SDGs in Turkey but also it introduces the relationships among the SDGs in the Turkish context. The relationships are categorized as affecting or being affected by a relevant SDG. The competencies of $\mathrm{OzU}$, the priorities of Turkey and the interactions among the SDGs are depicted in Figure 1 which also includes the direction of the interactions, i.e. affecting and being affected. In addition, in this figure the size of the SDG node determines the degree centrality of this specific SDG emphasizing its importance in the network of SDGs. The higher the size of the node of an SDG, the higher the degree centrality therefore the higher its importance for the sustainable development of Turkey.

In this analysis, the SDGs that are not Turkey's priorities or OzU's competences are not included for the sake of simplicity. Three SDGs are left out: SDG 3, 6, and 14. SDG 3 and 
SDG 14 are the goals that $\mathrm{OzU}$ is not competent in as the university does not have faculty or department in medicine or marine life. OzU is contributing to SDG 3 only with its Psychology Department both in terms of research and education. Since all the Turkish population has access to clean water and sanitation SDG 6 is regarded as completed hence not a priority for Turkey [9].

The [9] Report, also listed the inter-SDG interactions that are important for Turkey as well. In Figure 1, these interactions are depicted with dark blue. In addition, Turkey's priorities (in red rectangles) and the competencies of $\mathrm{OzU}$ (in blue rectangles) are illustrated.

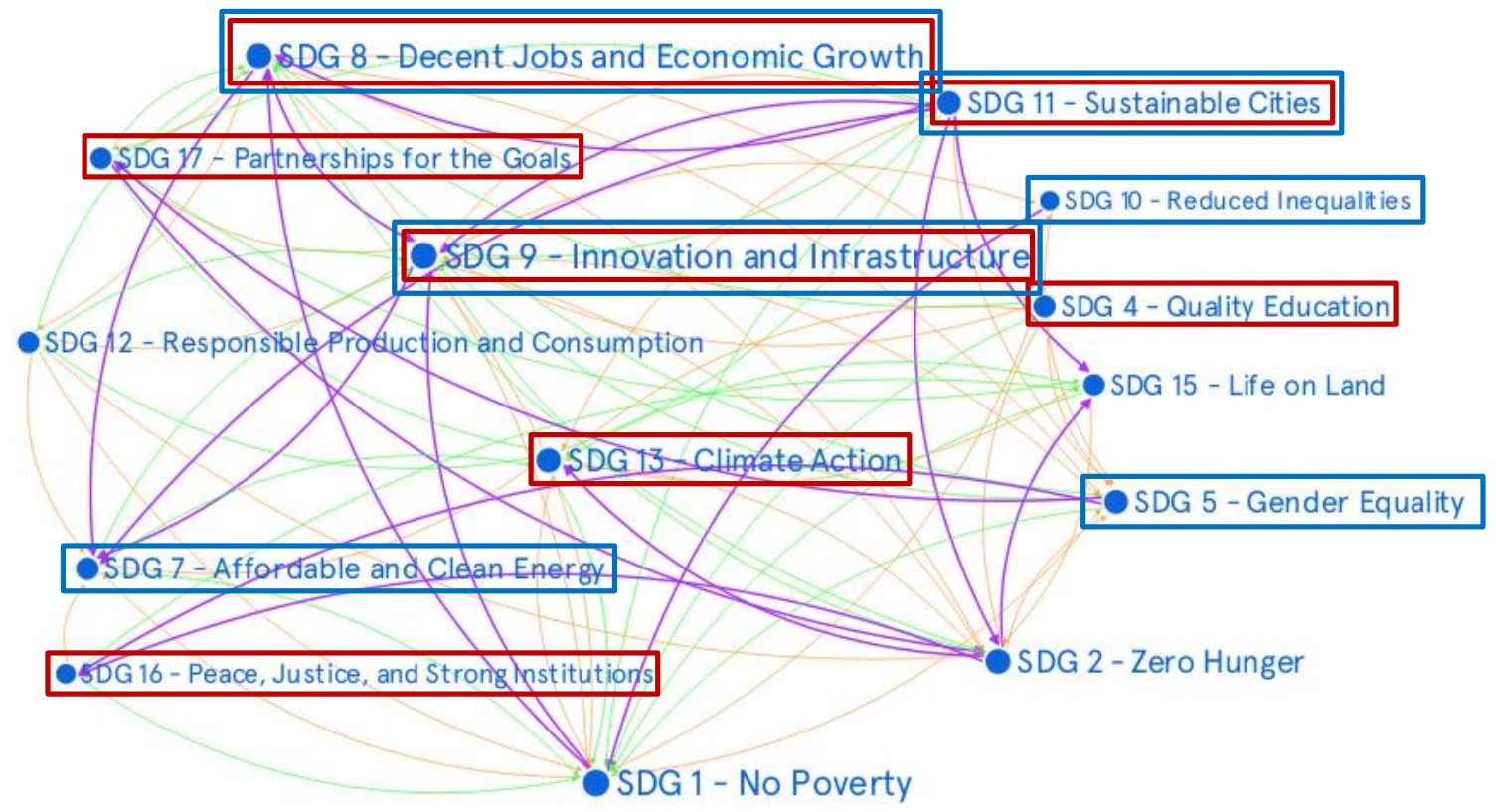

Figure 1. The interconnections among the SDGs.

At first glance, Figure 1 reveals 5 important SDGs: $8,9,11,13$, and 16 . SDGs 8 and 9 are the ones that are the most important two SDGs for Turkey to achieve sustainable development (see Figure 2). This is due to, first, their separate impacts are high and second, they are highly interlinked. In addition, SDG 8 and SDG 9 have high degree centrality, i.e. they both affect and are affected by various SDGs.

\subsection{The Sustainability Clusters}

This analysis led to the creation of four Sustainability Clusters, each of which led by a coordinator, the core of the structure that all the sustainability initiatives at $\mathrm{OzU}$ are managed through. Under each cluster are the SDGs that form a "cluster" by the intersecting Turkey's priorities, the interactions, and OzU's competencies. The clusters are presented in Figure 2.

As seen in Figure 2, every cluster has two types of SDGs: the primary and secondary SDGs. Primary SDGs are the core SDGs for the cluster whereas the secondary ones are the peripheral ones. The former SDGs are the ones in the first line under each cluster whereas the latter ones are depicted in the second line in Figure 2. 
1. Decent Work and Economic Growth

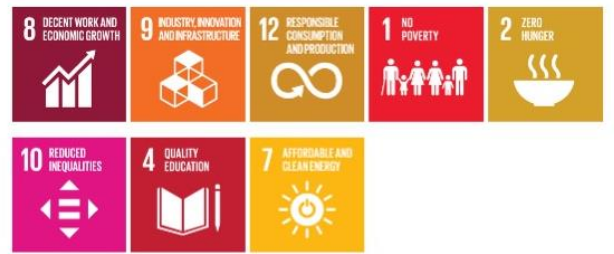

3. Energy and Climate

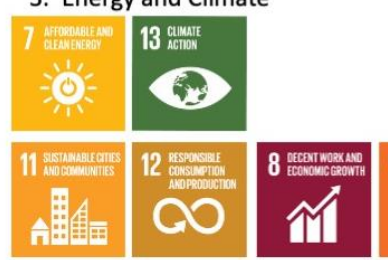

2. Sustainable Cities

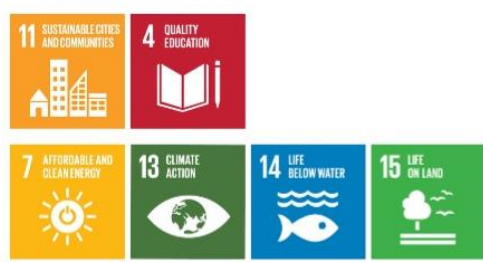

4. Social Equality

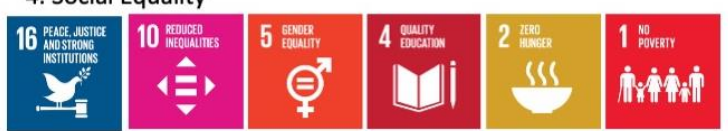

Figure 2. OzU Sustainability Clusters

The cluster structure is employed in essence to foster interdisciplinary initiatives. As Figure 1 illustrates all the SDGs are related with each other. Therefore, none of them can be tackled in isolation from the others. The analysis led to the clustering of the most interconnected SDGs with each other hence fostering interdisciplinary action. Moreover, the clusters themselves are not isolated from each other. Inter-cluster actions e.g. projects, research, etc. are highly sought after. As a matter of fact, cluster coordinators meet on a regular basis to both disseminate knowledge and identify and seize potential inter-cluster collaborations. Last but not least, SDGs under clusters are not static. This fluent structure allows the clusters to adopt to the changes that might occur in the SD landscape.

\subsubsection{SDG 8 and Sustainability Cluster 1}

The formation of only one cluster, Cluster 1, will be detailed here due to space restrictions. Other clusters are formed by using a similar method. Recall that interconnections among the SDGs are important for the formation of clusters. The interconnections of SDG 8 are presented in Figure 3.

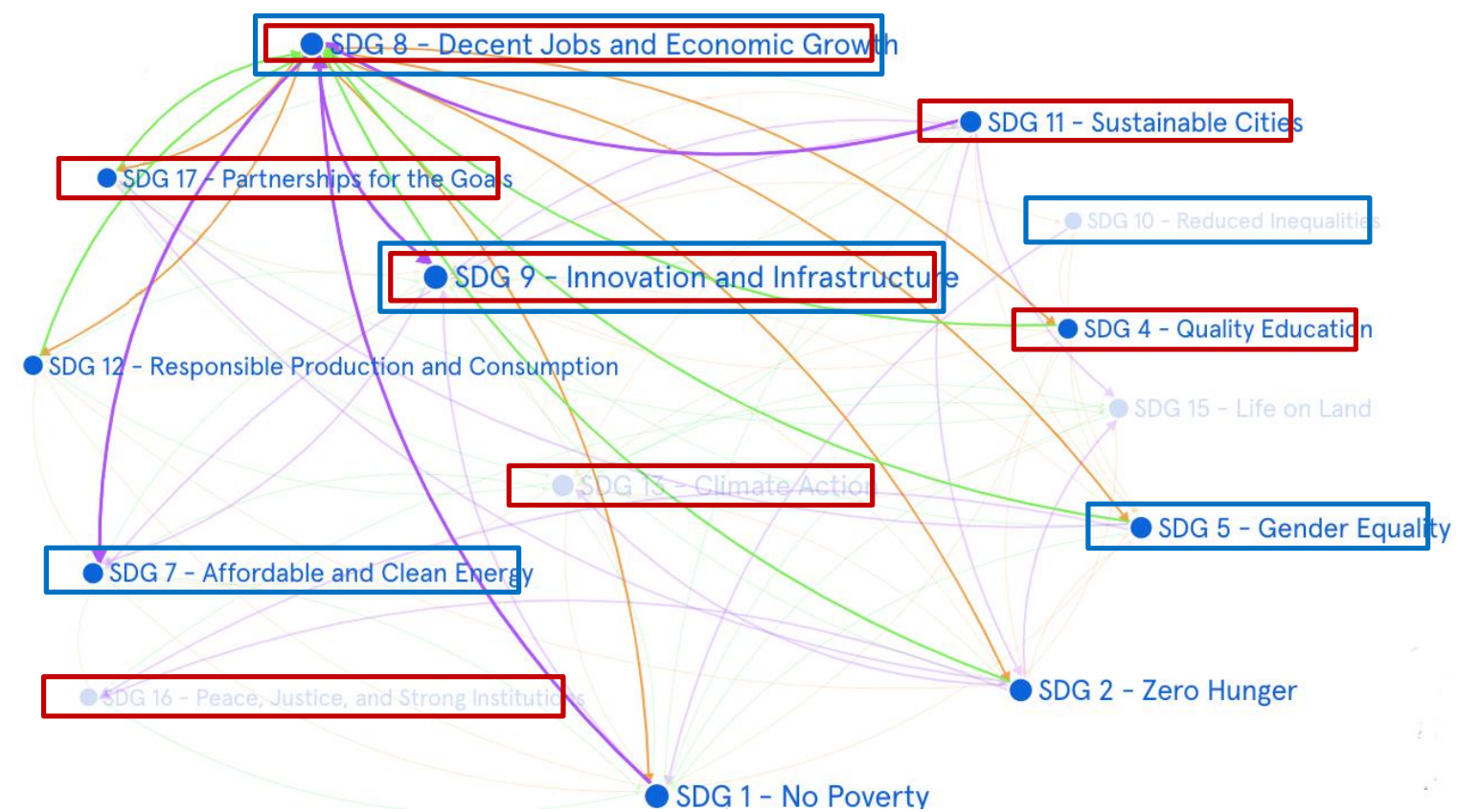

Figure 3. SDG interconnections focused on SDG 8. 
For Turkey and thus for Sustainability Cluster 1, the most important SDG is SDG 8. SDG 9 is the second most important SDG as it is paving way towards development. These SDGs both affect each other and $\mathrm{OzU}$ is competent in both. Figure 3 depicts the interconnections among the SDGs in this Cluster by focusing on SDG 8 with its interlinkages and priorities for Turkey, forming the basis of Cluster 1 together with SDG 9. Considering SDG 8's ties with first SDG 9 and subsequently with SDGs 1, 2, and 12 these three SDGs become the other primary SDGs. The secondary SDGs are SDGs 4, 7, and 10. Among these, SDG 8 is strongly related with SDG 7 however it is not included in this cluster as SDG 7 is the primary SDG of Sustainability Cluster 3.

\subsubsection{Other Clusters}

Although the creation of one cluster is detailed, the path should be clarified for other clusters. For Sustainability Clusters 2 and 3, the formation process is straightforward and follows that of Cluster 1 . For Cluster 4 , the tie between SDGs 16 and 5 is utilized. SDG 10 is added to the association making the focus of this Cluster inequalities of all types.

\subsection{The Overarching Body: Sustainability Platform}

Within this framework, to manage and coordinate all sustainability initiatives of the university a new unit is formed: OzU Sustainability Platform (SP). SP consists of dedicated staff as well as the coordinators of Sustainability Clusters. Together with the staff and coordinators, SP plays an active role in academic endeavors by identifying possible connections among disciplines fostering multidisciplinary research and teachings. Additionally, in cooperation with various units within the university SP helps maintain a sustainable university campus by developing and executing various projects. To support all these activities, SP creates coordinated networks to foster cooperation among stakeholders including faculty members, university staff, students, government bodies, and private sector both at the international and local level. Overall, SP is an integrated model with multidisciplinarity, participation, and inclusion.

\section{Concluding Remarks}

This paper provided the details of a strategic structuring process regarding the sustainability activities of Ozyegin University. The creation of the organizational structure initiated with self-assessment in which OzU determined the level competency it has in each SDG. Subsequently these strengths are aligned with the priorities of Turkey. Last but not least, taking into account the interconnections among the SDGs, OzU Sustainability Clusters are formed. Clusters are cooperative, fluent, and adaptive. With the addition of SP to the clusters, the sustainability management structure of $\mathrm{OzU}$ is formed. This structure is expected to foster interdisciplinary activities which in turn is expected to increase both the quality and the quantity of all sustainability initiatives by improving effectiveness and efficiency of these activities. However, since the structure is recently formed, the outcomes are yet to be observed.

One point to note is that, according to Figure 1, SDG 13 is one of the most important SDGs however it is not one of the SDGs that OzU is most competent at. Nevertheless, as the figure reveals SDG 13 has strong ties with various SDGs and all the sustainability related initiatives contribute to tackling SDG 13. 


\subsection{Practical Implications}

The path followed to create the cluster structure can be relevant for all type of organizations that aspire to contribute to the SD of their countries even if the resulting cluster structure may not be suitable for some organizations. OzU's experiences show that being aware of both the "self" and the context in terms of SD manifested in SDGs, makes the organizational design decision, if not all SD related decisions, effective and efficient. SDGs prove to be useful and clearly delineated tools to make such evaluations as well as making comparisons.

In addition, while designing these structures, it is important to refrain from creating rigid structures as the usually are rapid improvements in SD domain. For instance, during the design process the only tool that was present to understand Turkey was the report by [9]. However subsequently there have been many other reports as well as Voluntary National Reports. Furthermore, for instance, regarding the interactions among SDGs there are various developments including guidelines on how to determine SDG interactions [10, 11]. The organizational structures should be designed in a way to adapt to these developments in the field.

\section{References}

1. Fuso Nerini, Francesco, Julia Tomei, Long Seng To, Iwona Bisaga, Priti Parikh, Mairi Black, Aiduan Borrion, et al., 2018. Mapping Synergies and Trade-Offs between Energy and the Sustainable Development Goals. Nature Energy 3, 1, pp. 10-15.

2. Morse, Wayde Cameron, Max Nielsen-Pincus, Jo Ellen Force, and JD Wulfhorst, 2007. Bridges and Barriers to Developing and Conducting Interdisciplinary Graduate-Student Team Research. Ecology and Society, 12, 2.

3. Gardner, Susan K., 2013. Paradigmatic Differences, Power, and Status: A Qualitative Investigation of Faculty in One Interdisciplinary Research Collaboration on Sustainability Science. Sustainability Science 8, 2, pp. 241-52.

4. Brewer, Garry D., 1999. The Challenges of Interdisciplinarity. Policy sciences, 32, 4, pp. 327-37.

5. Golde, Chris M, and Hanna Alix Gallagher., 1999. The Challenges of Conducting Interdisciplinary Research in Traditional Doctoral Programs. Ecosystems, pp. 281-85.

6. Thurow, Amy Purvis, Charles William Abdalla, Julie Younglove-Webb, and Barbara Gray, 1999. The Dynamics of Multidisciplinary Research Teams in Academia. The Review of Higher Education 22, 4, pp. 425-40.

7. Lélé, Sharachchandra, and Richard B Norgaard, 2005. Practicing Interdisciplinarity. BioScience 55, 11, pp. 967-75.

8. Eigenbrode, Sanford D, Michael O'rourke, JD Wulfhorst, David M Althoff, Caren S Goldberg, Kaylani Merrill, Wayde Morse, et al., 2007. Employing Philosophical Dialogue in Collaborative Science. BioScience, 57, 1, pp. 55-64.

9. Escarus, 2017. Sürdürülebilirlik Hedefleri Kapsamında Türkiye'nin Mevcut Durum Analizi (Analysis of Turkey's Current Situation Based on Sustainable Development Goals).

10. Griggs, D., Nilsson, M., Stevance, A., and McCollum, D., 2017. A guide to SDG interactions: from science to implementation. (International Council for Science, Paris)

11. Nilsson, M., Griggs, D., and Visbeck, M., 2016. Policy: map the interactions between Sustainable Development Goals. Nature, 534, (7607), pp. 320-322 